\title{
A New Framework Simulation for Developing and Designing a Smart Campus Application
}

\author{
Mohammad Al Rawajbeh
}

\begin{abstract}
A framework simulation is proposed in this paper for developing a smart university application, incorporating an energy-efficient, sensor and actuator network, and an appropriate information integration methodology based on Semantic Web Service Composition. Real-time monitoring has been considered in this research along with the control of devices towards energy-awareness and optimization. The implementation of the proposed system could be useful in upcoming research where it includes the platform and evaluation of the equipment, network topology and ontology design, to exploit the services pertaining to the system in a most efficient, dynamic and fault tolerant way. Proper HumanComputer Interaction techniques has been employed in this research to provide end users with a user-friendly interface which will help in improving the "effectiveness" of university systems. The main goal of this project is to develop a proof-ofconcept $p^{1}$ rototype for a smart university system that will address some important challenges.
\end{abstract}

Index Terms - Smart buildings, IoT, smart university buildings, environmental monitoring, energy control, IoT applications.

\section{INTRODUCTION AND LETERATURE REVIEW}

The term 'smart buildings' refers to buildings empowered by the ICT, with sensors, actuators and embedded systems that allow to collect, filter and produce information to be exploited to provide functions and services [1].

In last two decades, the concept 'smart buildings' gained a great popularity related to the direct proportion of spread use of smart devices, actuators, intelligent devices and cloud computing. The main goal of building automation is the efficient and sustainable use of devices and components, comfort and control. In order to achieve sustainable performance and operations in smart building, several components must be taken into account such as the structure of buildings, technical systems and users as is offered in [2]. According to [3], smart building evolves into more than one connection across autonomous devices. It is leading towards systems and processes that are becoming more intelligent and capable of communicating with users.

The smart building definition as presented and defined in many references and researches is not limited to the remote controlling of the inner appliances, but to learning from the inhabitance behaviors. Additionally, it has the ability of taking the right decision itself and the ability to improve within the living environment [4]. In this context, smart systems should be able to follow the human design approach and use all modern technologies and smart solutions to ensure reliability, interactivity, flexibility and accessibility anytime and anywhere according to the network model. This is in order to improve sustainability and living environment [5]. As can be illustrated from Fig. 1, the major trend of growth relies on the number of smart devices and on the wide spread usage, and which addresses some serious challenges, structures and challenges but regarding a wider scope that encompasses a several number of buildings and smart cities [6].

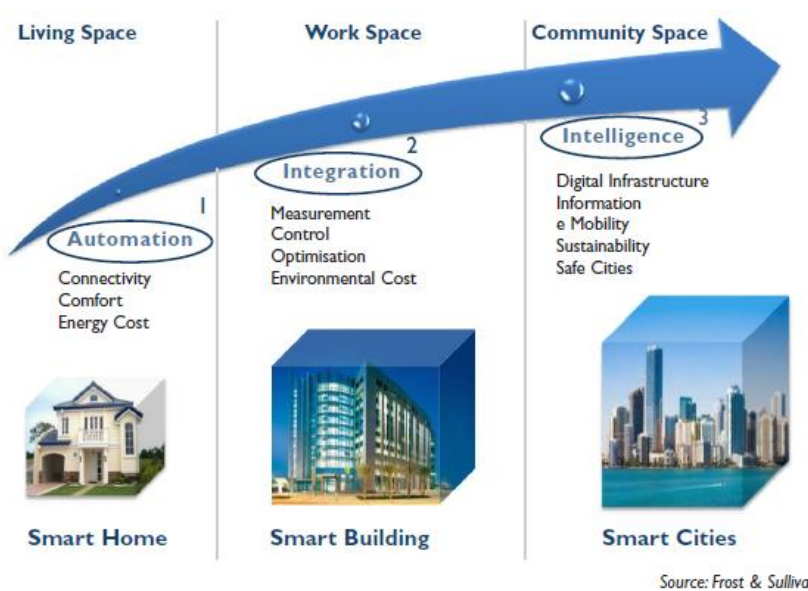

Fig. 1. The growth of smart devices usage.

Since the emergence of the smart building concept, many devices, interfaces and controllers have become available. The heterogeneity of different technologies, sensors, smart devices and other elements that are used in smart buildings are considered a problem for developing compatible and scalable smart home systems [7], [8].

In Ref. [9], a 4-layer system architecture is proposed for a large scale application for temperature monitoring. However, this structure is designed for complex systems as mentioned in [10]. A new methodology is reviewed by [11], which aimed at discussing the possibility of WSNs transitioning into modern IoT systems through the use of cloud computing technologies.

Nonetheless, the system faces difficulties with different interfaces, which means that APIs do not always exist.

\section{Problem STATEMENT}

The smart university buildings relies on information that is collected from different types of sensors such as light sensor, heat sensor, movement sensor and others environmental attributes sensors. The use of wireless communication network is considered as a critical part for
Manuscript received June 18, 2018; revised August 1, 2018

Mohammad Al Rawajbeh is with Faculty of Science and Information Technology, Department of Computer Science, Al Zaytoonah University of Jordan, Amman, Jordan (e-mail: m.rawajbeh@zuj.edu.jo). 
such technology by allowing the building management system to make a real-time control for the building environment through a communication network.

In small environments, smart devices and different sensors are connected into a point-to-point architecture, which in turn causes complexity for overall network system. In larger buildings, the use of wireless sensors, more smart devices, actuators, meters and control units make the installation of building's system bulky, complex, heavy and very expensive. Another side of this problem is the need for allocating material resources and efforts for installing and altering the system and sensors to acquire a full control of electronics in districts that are scattered and surrounding the university.

The potential problem in many proposed methodologies is that each component performs a single function and there is no synchronization with other components. A universal model is required to exploit the great potential of the new technologies that are employed by these devices. This universal model acts as a base for synchronizing devices, interfaces and intelligent control systems.

\section{THE PROPOSED PROJECT DESGN}

The main goal of this project is to connect several smart buildings inside the university territory. Such connection can be performed via information computer center where its main function is to have the full control of all domestics inside the buildings. Some examples include doors, windows ventilators, fire alarm. etc.

The main objectives of this project can be addressed as follows:

- Building Automation: In the smart university building environment, sensors and actuators are deployed spatially in the rooms of the building where monitoring and management are targeted. Many gateways serve as both sensors sinks and interoperability agents for various deployed sensor networks.

- Power Consumption: The most essential step in smart buildings is to reduction of the energy consumption. Hence, the implementation of the measuring and monitoring system is required. Some require that devices have to be installed for generating, measuring and monitoring the electricity in targeted buildings.

- Remote controlling systems: There are several remote controlling projects, which control smart devices remotely via the web or mobile messaging systems. The proposed simulator can connect to the server of these systems through a network or web. Users can observe the design that is simulated by using the Internet or a network, and can define a task to be conducted and then send it as a command to the remote controlling server.

- Supporting different types of sensors and actuators: There are many types of sensors, actuators and smart devices that can be used in modern buildings. The simulator which will be used for designing the smart campus building should have the ability to support different types of sensors and smart devices, and should be able to define the required details about their data type. It is important for in this paper to consider an effective and user friendly interface to facilitate the interaction between the user (admin) and the system.

\section{A. Project Requirements}

According to the earlier mentioned objectives, the project requirements is divided into two main parts, which comprise the hardware and software requirements.

- The hardware requirements: these requirements consist of several devices and unites depending on the level of functionality that is required in smart buildings. In this paper, smart buildings require the following parts (units):

1) Sensors: in this paper, different types of sensors must be used for sensing their environment in order to perform the functions of monitoring and submitting messages in case of any changes. Such sensors include smoke, gas, light and temperature sensors.

2) Actuators: these devices are working to perform the physical action depending on the case status, which is initiated on the base of the sensing process by some kinds of sensors such as opening doors, windows, switching light ON/OFF. etc.

3) Controllers: this unit is needed to make choices based on stored and programmed rules and instructions such as programs.

4) Central unit: rendering possible programs of units in the system (eg. registration server).

5) Communication network: supports the communication between different units and offers the users the required access to the system.

- The software requirement: The most appropriate simulation software for this paper is the new version of Cisco Packet Tracer 7.0. This simulator is considered one of the most powerful simulators. In particular, it contains new software tools that are needed in the paper such as sensors, actuators and smart devices including traditional communication devices.

\section{B. Proposed Project Structure}

The basic structure of the proposed smart campus is depicted in Fig. 2. The suggested platform is to design, develop and evaluate a smart building in a case study that involves Al-Zaytoonah University of Jordan (ZUJ) and that delivers the aforementioned objectives.

The structure of smart university consists of several parts. The physical part such as sensors, actuators and many other smart devices. The control system part, which works as an expert system since it decides what to do after some events taking place. The last part is the communication network part that connects the control system with physical devices using both wired and wireless communication technologies in order to have redundancies in channels.

In smart buildings, there are many sensors and actuators that are deployed in several rooms of buildings. In such structure, gateways are required to work as sinks for sensors and agents for interoperability between different types of sensor networks.

The monitoring and controlling processes are performed in the form of modular web services where the user can access the contents of building parameters based on devices connected to networks. The devices in buildings are based on the address technology, which will allow the user to access and be controlled by a server. In this paper, the control center of these devices is the computer center of the university. 


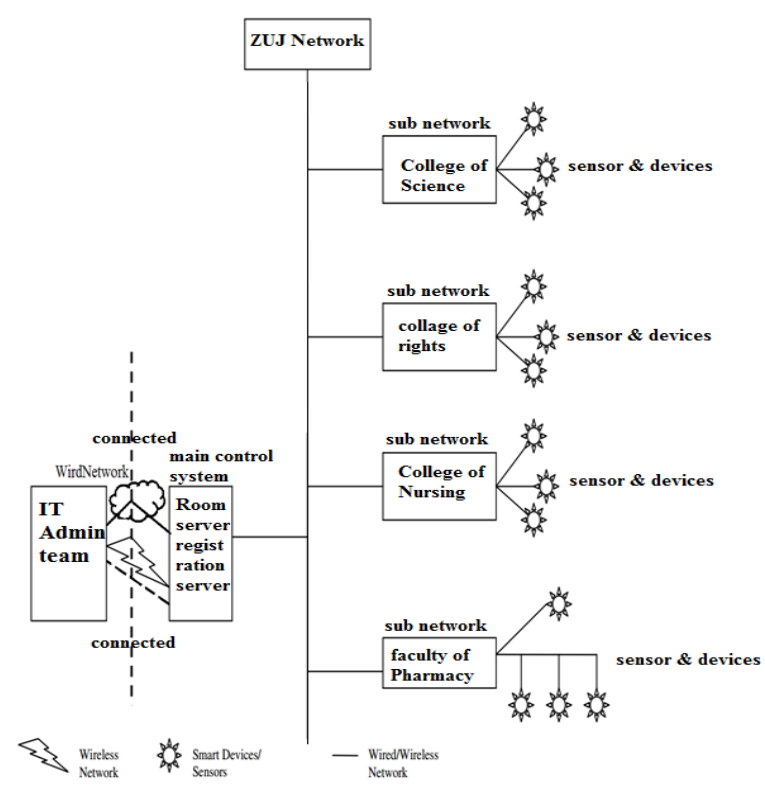

Fig. 2. The basic structure of a Smart ZUJ System.

The control system can be accessed by a computer central network of the internal network of the university buildings by using a mobile communication network, internet or a local network.

In such a system, the physical components (all kinds of sensors) sense the environment and collect the required information, and send them through the subnetworks to the computer central building of the university. Based on this information, the university control system takes the appropriate decision and sends the control information to the actuators through the network. For instance, the smoke sensor detects the smoke leakage in a smart building and passes this signal to the computer center through a wired network. The control system decides to either or not switch off the smoke valve and passes this to actuator, which will off/close the smoke valve or open the window.

\section{The Proposed Project Simulation}

The simulation process for designing the proposed project as shown in Fig 3 illustrates that the smart university system consists of many subsystems. Each subsystem consists of a huge number of components. The figure represents the system where all components and parts are integrated to each other by a communication network.

Fig. 3 illustrates the model of the proposed smart university project where this system consists of many physical components that have been bulged together and each component has its own function. According to the previous figure, it is found that the university consists of a few buildings where each of these buildings reflect each other for the part of the total system. Each of these buildings are summarized separately and argued by an existing hardware and function to be performed by each device and the internal connectivity among them.

\section{- The Faculty of Science and Information Technology Building}

It could be seen from Fig. 4 that the Science and Information Technology building and the smart devices are connected with each other. According to this figure, it can be shown that the number of smart devices could be controlled locally or remotely by using the PC or the smart phone based on an IP address and a logical program such as window, fan, door, smoke monitor, light, humidity LCD, water monitor, Lawn Sprinkler, Alarm, microcontroller (Arduino), Generic Environment Sensor, Hematuria Sensor, Solar Panel, Power Meter, Battery, Wind Turbine, Cell Tower and smoke source (old car). Some of these devices can turn on or off by controlling them locally or remotely. Other smart devices are considered dummy devices represented as sensor, each of them have program to act in specific events such as fire sensor to: turn the Fire Sprinkler on, alarm to: Warn employee or computer center of the presence of smoke within each university building, smoke sensor: to warning flags the presence of the amount of smoke after reaching a certain limit.

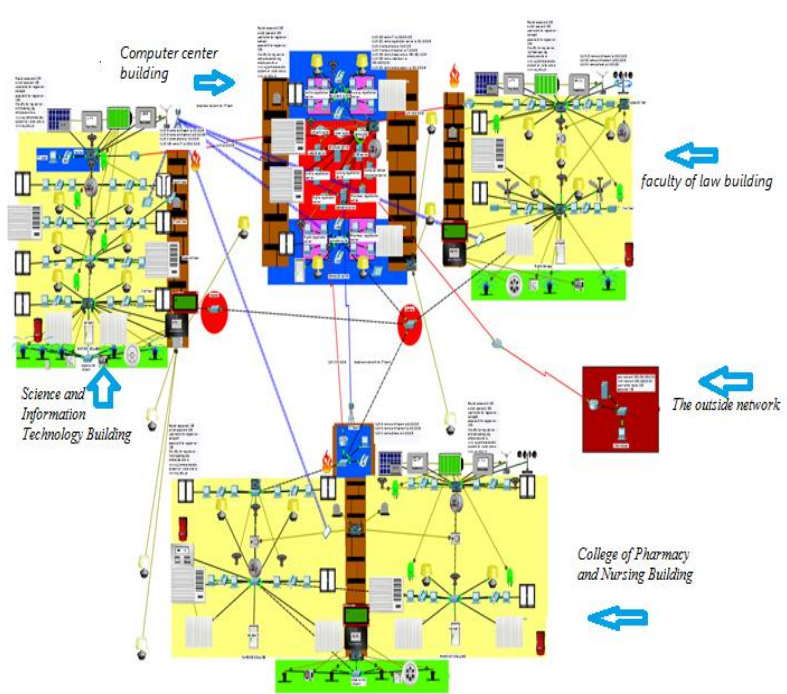

Fig. 3. The smart university design.

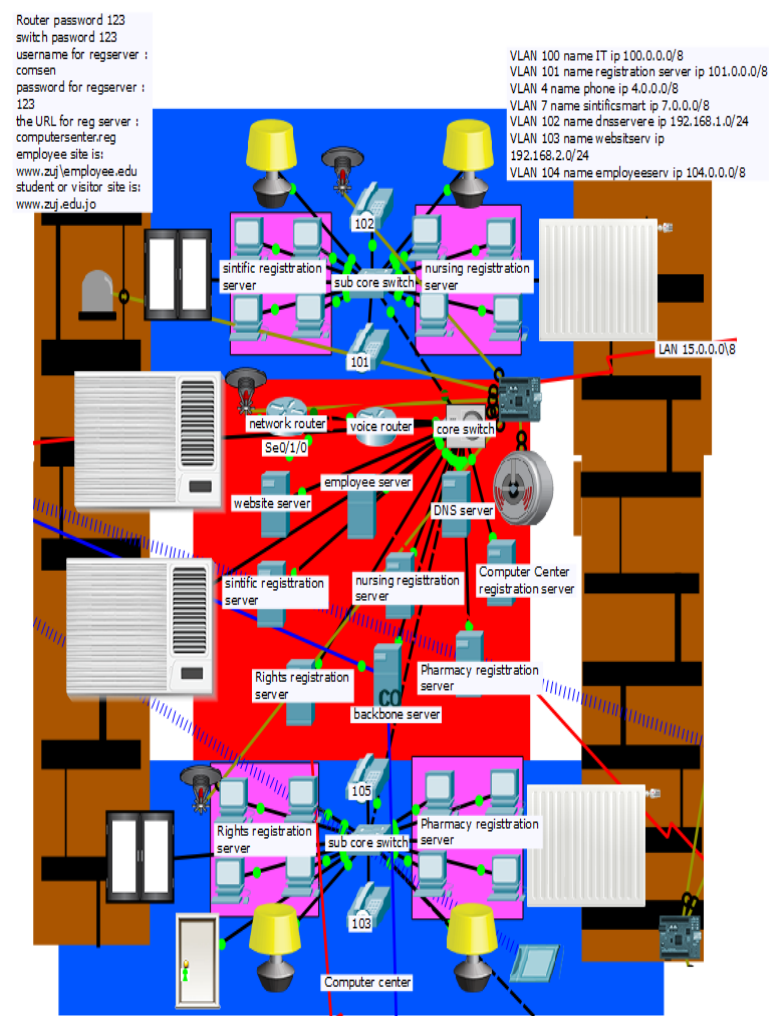

Fig. 4. The science and information technology building design.

In monitored or controlled smart devices, the IP address, username and password key are being used in order to 
manage the server registration.

\section{- University Computer Center Building}

Fig. 5 introduces the university computer center building and the smart devices that are connected together. According to this figure, the number of smart devices that will be controlled locally or remotely by using a PC or smart phone is explored. This can be performed by exploring the IP address and logical programs such as window, fan, door, smoke monitor, light, Alarm, microcontroller (Arduino). Some of these devices can be controlled by turning them on or off locally or remotely.

Other smart devices are dummy devices represented as sensor, each of them have program to act in specific events such as fire sensor to: turn the Fire Sprinkler on, alarm to: Warn employee or computer center of the presence of smoke in each of university building, smoke sensor: to warning flags the presence of the amount of smoke after reaching a certain limit. All these devices are connected with a control unit, namely, Arduino (MCU) to manage the program and send the alert at the right time. These devices are connected by an IOE custom cable.

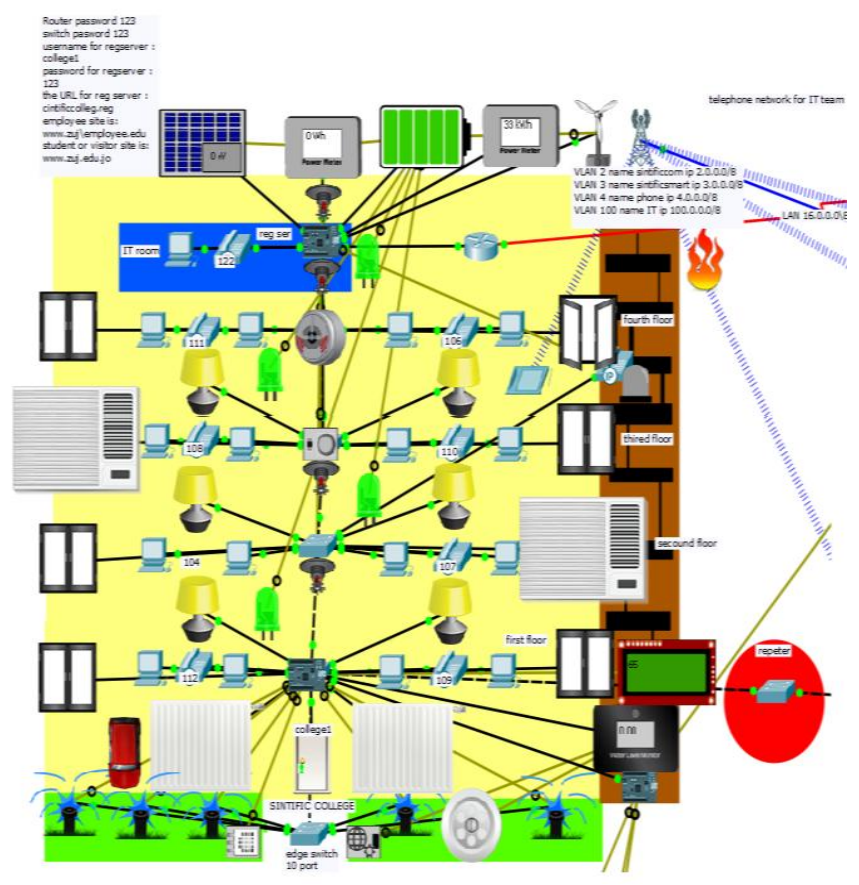

Fig. 5. The university computer center building design.

To control these smart devices and program, they are connected to switch the use of twisted pair cable.

In monitored or controlled smart devices, the IP address, username and password key are being used in order to manage the server registration.

\section{Registration Server Implementation}

The role of registration server in this paper is to register smart devices such as doors, windows, lights, etc. This function can be conducted on the server by using the Java program module. Based on this function, the registration server simulates the client-server concept where the smart device with the Java program module represents the client part.

The server, the user and the smart device are each assigned with an IP address. The address could be a static or dynamic address depending on the DHCP. Thus, the IP address will connect these elements with each other and facilitate the access to the smart device. After that, the smart device is defined on the registration server manually via the GUI, which resides on this smart device including the User name, IP server and Password.

The connection is initiated between the server and the smart devices by opening the connect page for the smart devices and creating an account. To create an account, inserting the username and the password that resides on the smart device is required, then the page is closed and reopened for this insertion. The result is a page containing the smart device, which can be controlled by the code that is residing on it as shown in Fig. 6. The smart device code can be reached by clicking on the device and by selecting it in advance. A tab with a required code appear and could be modified to make the smart device be able to be controlled by the user. It is important to mention that several accounts could be created in a server. The following figure highlights the process of creating an account and defining the smart device on a server.

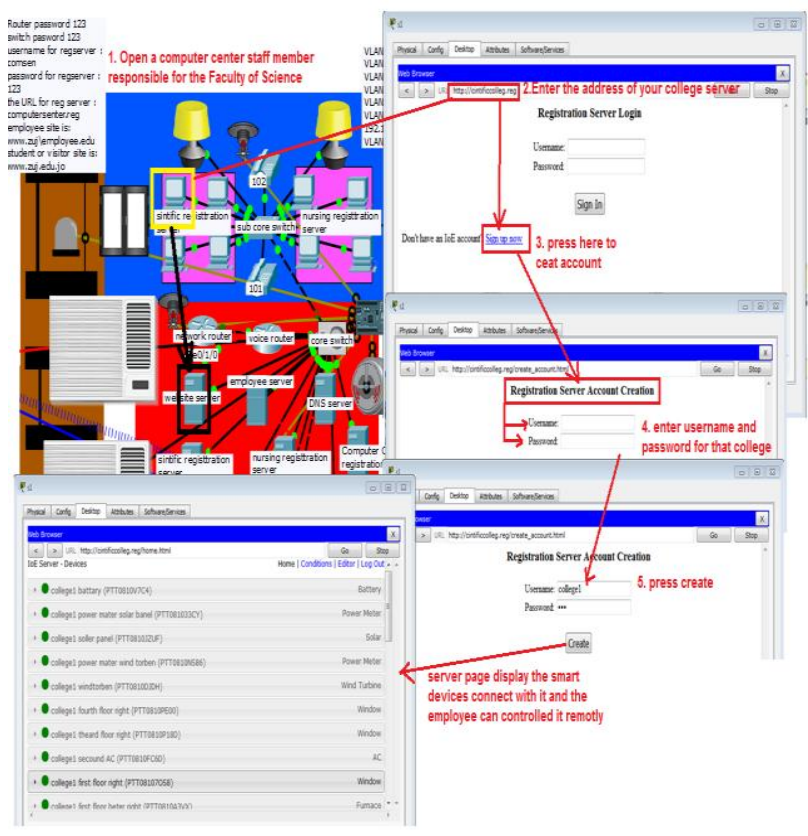

Fig. 6. Creating an account on the registration server.

\section{CONCLUSION AND FUtURE RESEARCH}

The proposed project in this paper presents a comprehensive framework for building a smart university application where different types of sensors and actuators are integrated in one integration methodology. A real time monitoring and controlling system are provided through different web services. In this project, smart devices are used with advanced network technologies. Such a methodology reduces the cost and increases the efficiency of the system. Additionally, this system could be able to work with different network services and servers by providing the main facilities for the automation, and power creation and conservation of the university buildings.

In terms of the future research, different variables and functions of this project should be monitored and studied in addition to some computations corresponding to the energy 
consumption for developing a real and logical plan to implement this project in reality.

\section{ACKNOWLEDGMENT}

The author would like to gratefully thank Al Zaytoonah University of Jordan for supporting this research project. The author would also like to acknowledge the efforts of some of our current undergraduates: Hamza, Khader, Rakan Al Ayyat and Omar Al Qahwaji for their efficient implementations of the prototype system under the author's supervision.

\section{REFERENCES}

[1] C. Bolchini, A. Geronazzo and E. Quintarelli, "Smart buildings: A monitoring and data analysis methodological framework," Building and Environment, 2017.

[2] F. Valentina, S. Giorgia, and P. C. Stefano, "Insights on smart home concept and occupants' interaction with building controls," in Proc. 8th International Conference on Sustainability in Energy and Buildings, Sep. 2016, pp. 759-769.

[3] G. M. Toschi, L. B. Campos, and C. E. Cugnasca, "Home automation networks: A survey," Computer Standards \& Interfaces, vol. 50, pp. 42-54, 2017.

[4] I. B Eugeny, "The distinctive features of smart buildings," in Proc. $X I V R-S-P$ Seminar, Theoretical Foundation of Civil Engineering (24RSP) (TFoCE 2015), 2015, pp 103-107.

[5] M. Coccoli, A. Guercio, P. Maresca, and L. Stanganelli, "Smarter universities: A vision for the fast changing digital era," Journal of Visual Languages and Computing, vol. 25, pp. 1003-1011, 2014.

[6] P. Vijaia and P. B. Sivakumar, "Design of IoT systems and analytics in the context of smart city initiatives in India," in Proc. 2nd International Conference on Intelligent Computing, Communication \& Convergence, Procedia Computer Science, 2016, vol. 92, pp. 583 588 .
[7] M. Al Rawajbeh, "Low cost design and implementation for HAS using multifunctional Wi-Fi," International Journal of Computer Networks \& Communications (IJCNC), vol. 9, no. 3, May 2017, pp. 105-116.

[8] M. Al Rawajbeh, "Advanced object monitoring using wireless sensors network," in Proc. International Conference on Communication, Management and Information Technology, Procedia Computer Science, 2015, vol. 65, pp. 17-24.

[9] Q. Zhang, Y. Wang, G. Cheng, Z. Wang, and D Shi, "Research on warehouse environment monitoring system based on wireless sensor network," in Proc. 2014 9th IEEE Conference on Industrial Electronics and Applications, pp. 639-1644.

[10] W. Li and S. Kara, "Methodology for monitoring manufacturing environment by using wireless sensor networks (WSN) and the internet of things (IoT)," in Proc. the 24th CIRP Conference on Life Cycle Engineering, Procedia CIRP 61, 2017, pp. 323-328.

[11] K. Ahmed and M. Gregory, "Integrating wireless sensor networks with cloud computing," in Proc. Seventh International Conference on InMobile Ad-hoc and Sensor Networks, 2011, pp. 364-366.

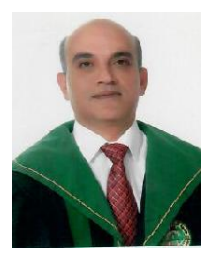

Mohammad Al Rawajbeh is an associate professor at the Computer Science/Computer Networks Department, Faculty of Science and Information Technology, AL Zaytoonah University of Jordan. He received the High Diploma Degree in Computer Technology from Yarmouk University, Jordan in 1991; B.SC and master degree in computers and networks From Kharkov National Polytechnic University, Ukraine from 1992 to 1996 . He obtained his Ph.D. degree in computer network management from Kharkov National University of RadioElectronic, Ukraine in 2000. His research interests are in the fields of network management, performance evaluation, and internet technology and wireless sensor networks. He has more than 20 published articles in International Journals and Conferences. 